\title{
3D scan analysis as a tool for comparative study of coverall design
}

\author{
Susan Ashdown, Fatma Baytar, and Anita Mbogoni, Cornell University, USA
}

Keywords: 3D body scanning, coverall, fit analysis, product development

Introduction. Pesticide protective coveralls (PPCs) are worn by agricultural workers to prevent harmful chemicals from coming into contact with the worker's skin and clothes. PPCs are onepiece and unisex garments, typically with a hood and zippered front, and must fit a wide range of body types (Keeble, Prevatt \& Mellian, 1992). They are usually made from non-woven fabrics that are chemical repellent, but are prone to rips when worn, generally because of fit issues. These coveralls are meant to protect the worker while allowing them to exercise the full range of motion necessary to complete agricultural tasks such as stepping up into a tractor and reaching to pick crops above their head. If workers do not have properly fitting PPCs, their safety is at risk because of badly designed and sized coveralls may both restrict movement and expose the worker to contamination through tears (Depa, 2016). Moreover, any fit or design problems in PPCs were found to negatively affect the wearer's perception of the level of protection offered by the garment (Boorady et al. 2009). Three-dimensional (3D) scanning technology enables the creation of digital models and allows for a wider scope of observation to identify fit problems in garments. Therefore, this study explores the use of 3D scanning technology to identify fit problems in PPCs. More specifically, objectives of this study were to (a) test and compare the fit of three coverall designs to see which ones promote comfort and do not restrict movement, and (b) determine sizing and fit problems in coveralls.

Methods. Participants were recruited through flyers posted in various locations around a university campus, postings on university social media webpages, and through emails sent to work groups (e.g., greenhouse workers). Most of the respondents were undergraduate students at the university. When participants arrived in the lab for scanning, their height and weight measurements were taken to assign them to suggested PPC sizes that correspond to an individual's height. A Human Solutions Vitus XXL 3D body scanner was used for data collection. Participants tried on three coverall designs: two commercial (PPC1 \& PPC2) and one innovative design by the research group (PPC3). Each participant was scanned in five poses (one standing, and four active) in each of the three coveralls, and in the same five poses minimally clothed. Minimal clothing, worn over the participant's own underwear, comprised a form fitting sleeveless top and shorts for female participants and a pair of boxer briefs for males. Active poses were set using a goniometer for consistency. The hoods were worn up on the head, and the coveralls were taped to gloves at the wrist and taped to the ankle. After the scanning sessions,

Page 1 of 3

Published under a Creative Commons Attribution License (https://creativecommons.org/licenses/by/4.0/), which permits unrestricted use, distribution, and reproduction in any medium, provided the original work is properly cited.

ITAA Proceedings, \#76 - https: / /itaaonline.org 
participants rated ease of movement and overall fit in each coverall and posture by using a 5point Likert scale ( $1=$ very difficult/uncomfortable and $5=$ very easy/comfortable). They were also asked to comment on their favorite and least favorite features of each coverall, and on any features that they thought could be improved.

Results. A total of 50 males and females agreed to participate in the study. Of the 50 participants, $30 \%(n=15)$ were male and $70 \%(n=35)$ were female. Participants ranged from 18 to 44 years, with a mean of 23 years. The mean height of participants was 168.86 centimeters and the mean weight was 65.34 kilograms. $40 \%$ of participants $(n=20)$ had previous experience wearing protective coveralls; of these, six had previous experience wearing coveralls for pesticide application. Even though participants were provided with the sizes that, based on the coverall sizing chart, corresponded to their height; the most common fit problem reported across all three coveralls was that size provided was too large. This was especially reported frequently in both of the commercial coveralls, which had excess fabric that left space between the coverall and the body (Figure 1a, 1b). The innovative design, by contrast, laid closer to the body. Eight participants indicated disproportionate fit in areas of PPC1. Criticisms included legs too long for the participant, leading to bunching at the bottom of the leg; and a low crotch (Figure 1a), which created difficulty especially in taking active poses.

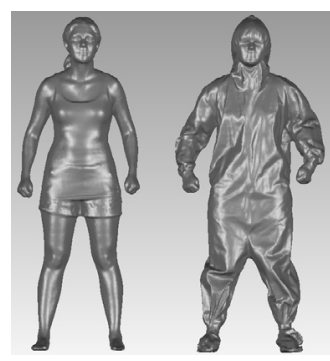

(a)

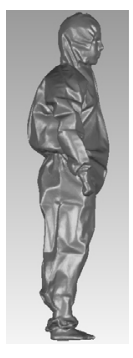

(b)

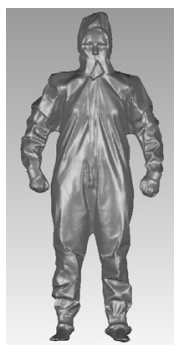

(c)

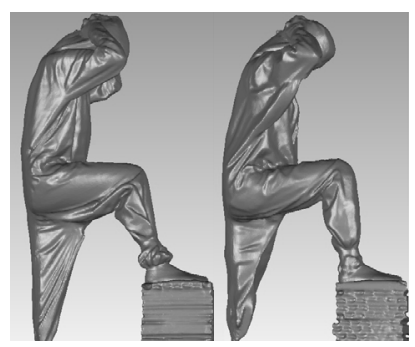

(d)

Figure 1. (a) Low crotch in PPC1 (right); same participant minimally clothed (left); (b) excess fabric in PPC2 (side view); (c) hood tightness in PPC3; (d) example of a difficulty with high stepping pose while wearing PPC1 (left); same participant wearing PPC3 (right).

Two participants experienced tearing with PPC1 during scanning sessions and indicated that the limited range of motion that lead to the tearing - and exposed parts of their bodies to the environment - would be unacceptable while performing agricultural duties. The tearing was not evident in the scans of these participants. However, as compared to PPC2 and PPC3, the crotch of PPC1 was visibly too low when worn by several participants. This finding suggested that PPC1 was at risk for tearing while being worn, and it could benefit from a redesign (such as shortening torso) to accommodate and ensure safety for wearers of varying heights. The most

Published under a Creative Commons Attribution License (https://creativecommons.org/licenses/by/4.0/), which permits unrestricted use, distribution, and reproduction in any medium, provided the original work is properly cited.

ITAA Proceedings, \#76 - https://itaaonline.org 
common fit problem reported for PPC3 was tightness in the hood area (Figure 1c), which led to difficulty moving the head and obscured visibility; about one fifth of participants reported that this was the feature they thought could be most improved. One of the active poses, i.e. the high stepping pose, was difficult for the highest number of participants, with 16 participants either unable to assume the posture at all, or only able to do so with some difficulty. Of these, ten indicated difficulty in assuming the posture while wearing PPC1, five while wearing PPC2, and one while wearing PPC3 (Figure 1d).

Conclusion. This study explores the use of 3D scanning in coverall design analysis, by comparing fit problems present in three different coverall designs from the marketplace and an experimental design. These problems were both reported by participants in questionnaires, and visually observed by the researchers in the resultant 3D models. The 3D scans allowed the researchers to observe fit problems that were not mentioned by participants, and to affirm participant criticisms of each coverall design. Results indicated that PPC1 was the least favored of the three, followed by PPC2, and then PPC3. Each of the three coverall designs tested in this study had at least one feature that was not favorable for some wearers, and that could benefit from a partial redesign to modify these features in order to better accommodate wearers. Additionally, all of the coveralls would improve if there were variations of each that were designed for different body types (e.g., medium coveralls that were available in both the standard length and tall length). This would allow for a better fit for wearers who are currently unable to find coveralls that fit well, and would give wearers improved comfort and protection in their protective clothing.

This material is based upon work that is supported by the National Institute of Food and Agriculture, U.S. Department of Agriculture, Hatch/Multi State under \#1017350.

\section{References}

Keeble, V., Prevatt, M.B., \& Mellian, S.A. (1992). An evaluation of fit of protective coveralls manufactured to a proposed revision of ANSI/ISEA 101, In Performance of protective clothing fourth volume. DOI: 10.1520/STP19196S

Depa, T.H. (2016). Trends in ... women's PPE. Outfitting the entire workforce. Safety + Health. Retrieved from: http://www.safetyandhealthmagazine.com/articles/14708-trends-inwomens-ppe

Boorady, L. M., Rucker, M., Haise, C., \& Ashdown, S.P. (2009). Protective clothing for pesticide applicators: A multimethod needs assessment, Journal of Textile and Apparel, Technology and Management, 6(2), 1-17.

Page 3 of 3

(c) 2019 The author(s). Published under a Creative Commons Attribution License (https://creativecommons.org/licenses/by/4.0/), which permits unrestricted use, distribution, and reproduction in any medium, provided the original work is properly cited.

ITAA Proceedings, \#76 - https://itaaonline.org 